\title{
Inappropriate medical management of spinal epidural abscess
}

\author{
P Harrington, P A Millner, D Veale
}

\begin{abstract}
A 67 year old man with longstanding rheumatoid disease was referred to the regional spinal surgery unit with acute onset of paraparesis due to an extensive spinal epidural abscess of the lumbar spine. Ten months previously, he had started antibiotic treatment at another hospital for an epidural abscess arising at the level of the L2-3 disc space. Despite completing seven months of medical treatment with appropriate antibiotics, he had a recrudescence of acute back pain shortly after restarting methotrexate treatment. Urgent anterior spinal decompression with excision of the necrotic vertebral bodies of L1-3 was performed. The indications for the surgical management of spinal epidural abscess are reviewed. (Ann Rheum Dis 2001;60:218-222)
\end{abstract}

Infection in the spinal epidural space is an uncommon but potentially fatal condition that
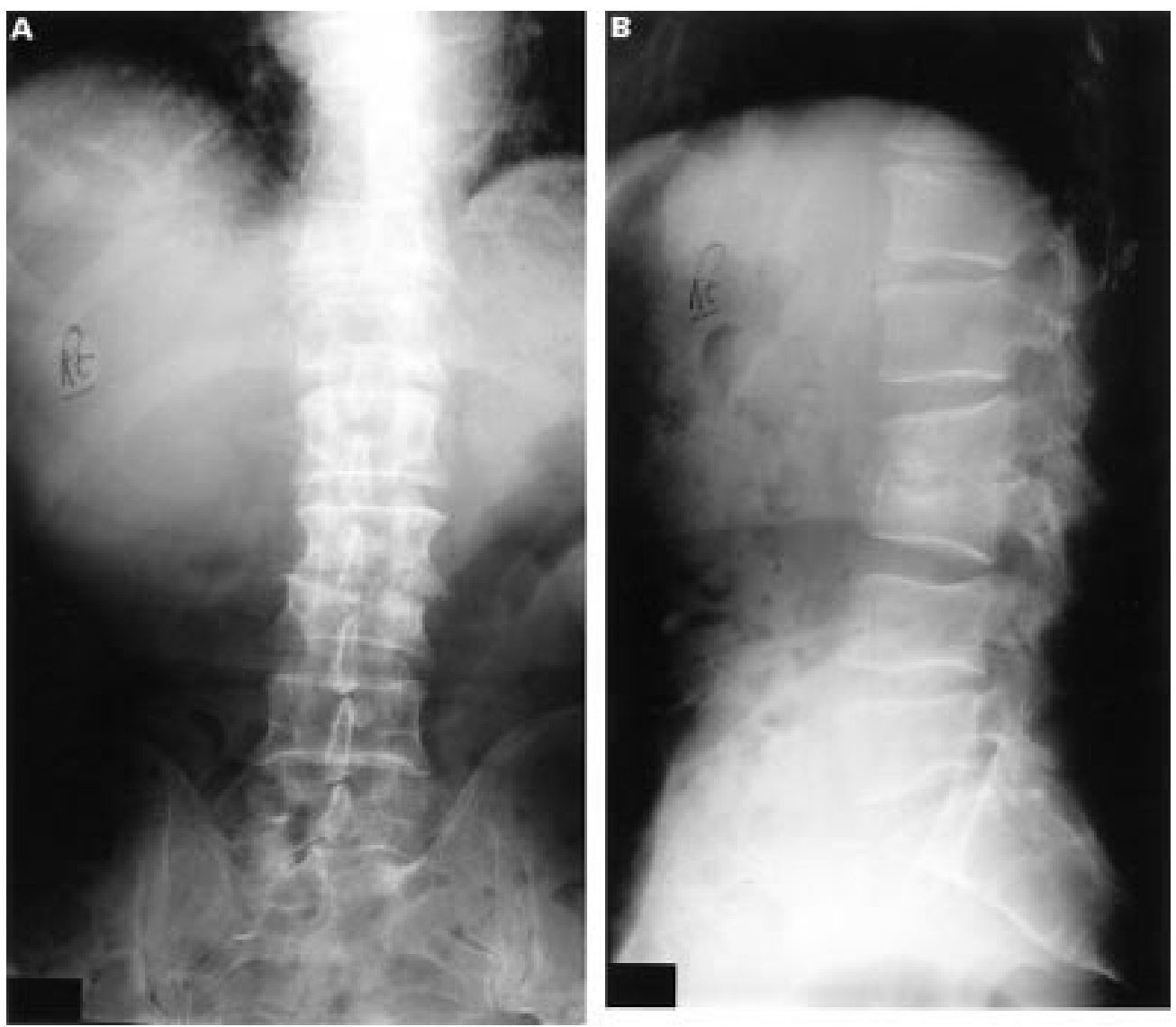

Figure 1 (A) Anteroposterior and (B) lateral radiographs of the lumbar spine at the time of initial presentation, showing destruction of the L2-3 disc space.

often constitutes a surgical emergency. Seventy eight per cent of patients have an identifiable source of infection, and specific risk factors such as diabetes, intravenous drug abuse, and immunosuppression are reported in $51 \%{ }^{1}$ Although Barth reported the first successful operation for spinal epidural abscess (SEA) in $1901,{ }^{2}$ early reports found that the condition was nearly always fatal, and in most cases diagnosis was only made at necropsy. ${ }^{3}$ The advent of antibiotic treatment and the acceptance of the necessity for surgical debridement improved the prognosis. ${ }^{4}$ Despite these advances, the mortality remains high, ranging from 7 to $18 \%$ in modern series. ${ }^{56}$

Before the availability of modern imaging techniques, surgery was performed on the basis of neurological and myelographic findings. Now, with computed tomography (CT) and magnetic resonance imaging (MRI), the diagnosis of SEA is routinely made much earlier. As a result of the early introduction of appropriate antibiotics, patients with SEA have been cured

Spinal Surgery Unit, St James's University Hospital, Leeds, UK

P Harrington P A Millner

Department of Rheumatology, Leeds General Infirmary, Leeds, UK

$\mathrm{D}$ Veale

Correspondence to: Mr P Harrington, 119 Castle Road, Hull HU16 5JF
Harrington@aol.com 
Table 1 Course of the laboratory indices (erythrocyte sedimentation rate (ESR), white cell count (WCC)) after initial presentation

\begin{tabular}{|c|c|c|c|c|c|c|c|c|}
\hline Date (1998) & 5 Jan & 27 Jan & 5 Mar & $7 \mathrm{Apr}$ & $27 \mathrm{Apr}$ & 16 Jun & $12 \mathrm{Oct}$ & $4 \mathrm{Dec}$ \\
\hline $\operatorname{ESR}(\mathrm{mm} / 1 \mathrm{st} \mathrm{h})$ & 40 & 110 & 100 & 94 & 122 & 110 & 60 & 96 \\
\hline $\mathrm{WCC} \times 10^{9} / 1$ & 7.9 & 7.8 & 28.7 & 14.7 & 16.2 & 12.6 & 8.7 & 25.1 \\
\hline
\end{tabular}

Note: Percutaneous drainage of paraspinal collection 30 January 1998.

Intravenous vancomycin discontinued 27 February 1998.

Septic arthritis knee joint 9 March 1998.

Teicoplanin discontinued, methotrexate restarted 12 October 1998

with medical treatment alone, and some workers have challenged the primacy of surgical debridement. $^{7-9}$

We report a patient with an extensive spinal epidural abscess and gross destruction of his lumbar spine who had undergone 10 months of unsuccessful medical treatment before referral to a spinal surgery unit.

\section{Case report}

A 67 year old man with a 10 year history of rheumatoid disease was referred to the regional spinal surgery unit at St James's University Hospital, Leeds, with acute onset of paraparesis due to an extensive spinal epidural abscess of the lumbar spine.

Ten months previously he had presented to the rheumatology service of his local hospital with cellulitis complicating chronic plantar ulcers on both feet. These ulcers had developed two months before and had been treated conservatively. The drugs used before admission included methotrexate $7.5 \mathrm{mg}$ weekly, folic acid $5 \mathrm{mg}$ daily, diclofenac $75 \mathrm{mg}$ twice daily, ranitidine $150 \mathrm{mg}$ twice daily. Wound swab cultures grew pseudomonas and methicillin resistant Staphylococcus aureus (MRSA). The erythrocyte sedimentation rate (ESR) was $90 \mathrm{~mm} / 1 \mathrm{st} \mathrm{h}$ and the white cell count (WCC) $9.7 \times 10^{9} / 1$.

Five days later, on 23 January 1998, the patient developed acute low back pain. Plain radiographs of the lumbar spine at that time showed destruction of the L2-3 disc space (fig 1). CT confirmed a spinal epidural abscess, extending along the psoas sheath. On 30 January 1998 percutaneous drainage of the paraspinal collection was performed under CT guidance. Twenty millilitres of pus was aspirated from the L2-3 disc space, which on culture grew MRSA with the following sensitivity spectrum: vancomycin, teicoplanin, gentamycin, rifampicin, fusidic acid. Intravenous vancomycin treatment ( $1 \mathrm{~g}$ daily) was started with close monitoring of the serum peak and trough levels because of problematic renal compromise. Two weeks after admission a general surgical opinion led to an open procedure to debride the psoas collection using a retroperitoneal approach through a left flank incision. Postoperatively, the patient had a pulmonary embolus and his clinical condition continued to deteriorate.

On 27 February 1998, intravenous vancomycin treatment was stopped because of the development of antibiotic associated colitis. Stool culture confirmed a diagnosis of pseudomembranous colitis secondary to colonisation with Clostridium difficile. Oral vancomycin treatment was started to treat this complica- tion. Ten days later the patient spiked a pyrexia $\left(38.5^{\circ} \mathrm{C}\right)$ and developed an acute inflammation of the left knee joint. Blood culture again grew MRSA and knee joint aspiration confirmed MRSA septic arthritis. The antibiotic sensitivities were as before. Intravenous teicoplanin $(200 \mathrm{mg} /$ day $)$ and gentamycin $(80 \mathrm{mg}$ twice daily) were started to treat this episode of MRSA septicaemia. Throughout this period the laboratory indices (ESR, WCC) remained persistently raised (table 1 ).

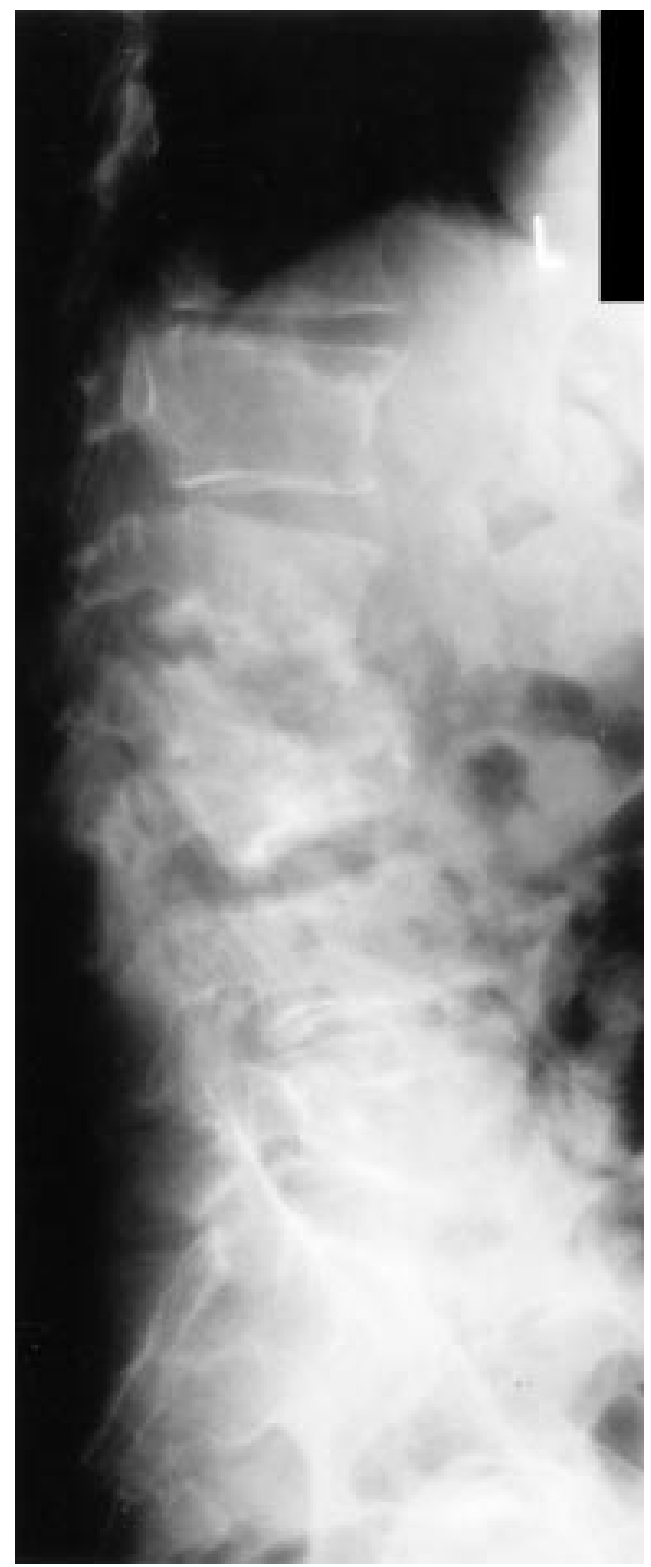

Figure 2 Lateral radiograph at time of referral to spinal surgery unit, 10 months after presentation, showing complete destruction of L2, most of L3, and the inferior end plate of L1. There is an obvious angular kyphosis at L2. 


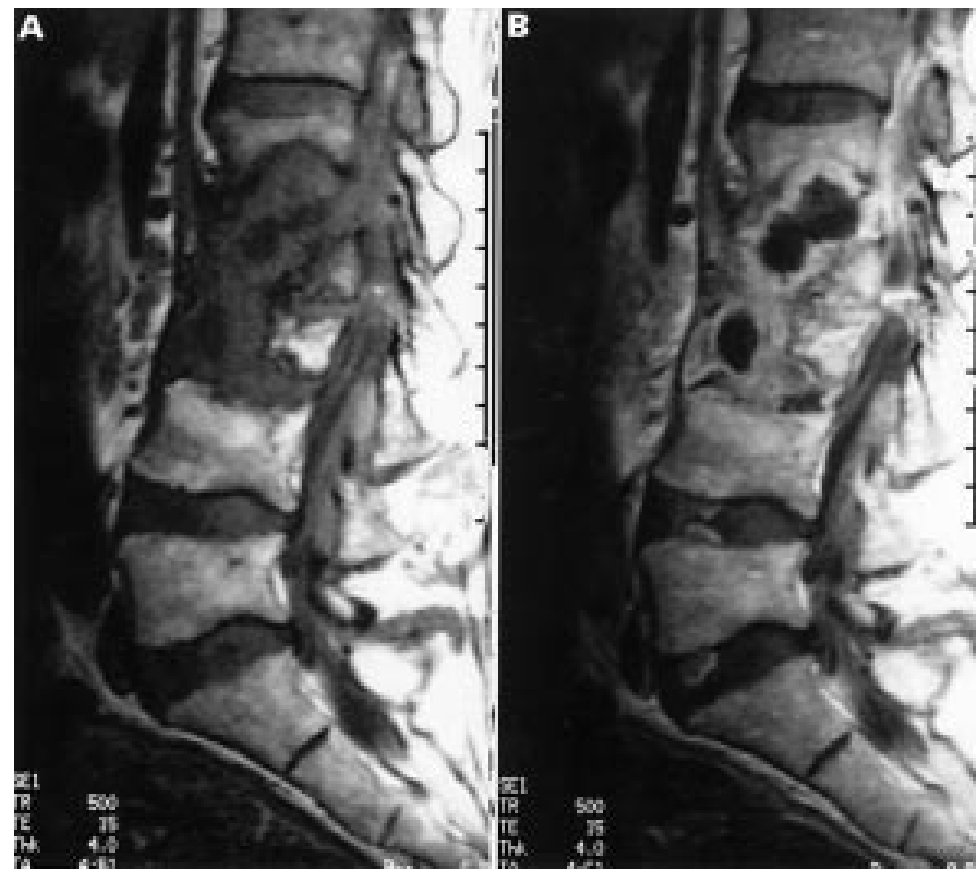

Figure $3 T 1$ weighted sagittal magnetic resonance imaging of the lumbar spine (A) before and (B) after gadolinium. Three large abscess cavities show rim enhancement with gadolinium. The bony destruction is more severe than the plain radiographs indicate, with involvement of the superior end plate of $L 4$.

Six weeks after admission his weight had fallen to $49 \mathrm{~kg}$ and he had developed pressure sores over the sacrum and left heel. Slowly, however, his condition improved, his family were instructed how to give domiciliary intravenous antibiotics, and he was discharged after a hospital stay of six months, walking independently.

Two months later he attended the outpatient department with acute pain and stiffness in the right ankle. Needle aspiration of the joint was negative. Antibiotic treatment was discontinued and methotrexate and anti-inflammatory drugs were reintroduced to treat this rheumatoid flare.

One month after that the patient presented to the accident and emergency department with a recrudescence of acute low back pain. Blood cultures confirmed MRSA septicaemia. The organism remained sensitive to teicoplanin. Plain radiographs of the lumbar spine confirmed extensive destruction (fig 2). The patient required urinary catheterisation for acute retention and was referred to the regional spinal surgery unit -10 months after the initial presentation.

On arrival at our unit, the patient was alert and oriented. He had a pyrexia and was markedly cachectic. He was paraparetic with reduced motor power, MRC grade $2 / 5$, in both legs and with a lax anal sphincter. MRI showed extensive destruction of the lumbar spine extending over three vertebral segments (fig 3 ), with pus in the epidural space tracking up to the upper thoracic level. After discussion of the considerable surgical risks in such a setting, the patient elected to proceed with surgical debridement. Preoperatively, a vena caval filter was inserted and warfarin treatment was discontinued. Anterior spinal decompression and cor- pectomy of levels L1-3 was successfully performed. Reconstruction was achieved using fibular strut grafts and Kaneda instrumentation (fig 4). Despite developing a left basal pneumonia postoperatively, the patient made an otherwise uncomplicated recovery and left hospital two months later (13 months after his initial presentation).

At a six month follow up visit the patient had no back pain and continued to live independently. He was able to walk with the aid of one walking stick.

\section{Discussion}

Reports of SEA have two common threadspoor outcome and appeals for earlier recognition and treatment to avoid same. However, the diagnosis of spinal abscess can be difficult because of its rarity and the insidious presentation. The significant associated morbidity and mortality are related to the elusive nature of the condition until it has reached an advanced stage. The reported incidence is one to two cases per 10000 hospital admissions and therefore many non-spinal specialists will never see a case. ${ }^{10}$

The standard management of SEA has been surgical debridement, with or without operative stabilisation, followed by prolonged antibiotic treatment. ${ }^{11}$ Numerous reports have appeared recently describing a non-surgical therapeutic approach. Wheeler et al reported a case of extensive SEA successfully treated without surgery. ${ }^{8}$ They reviewed the literature and documented 38 cases that were managed non-operatively in a 20 year period until 1990. They concluded that the literature did not support the widespread use of non-surgical management. Several authors have proposed guidelines for appropriate medical management. Del Curling and colleagues predicted in 1990 that non-operative management would be proved to be appropriate in patients with lumbar infection without neurological compromise, and in whom an infecting organism had been successfully identified. ${ }^{5}$ Baker et al listed the same guidelines and added the rider that if the temperature, WCC, and ESR all continued to decrease, then continued medical treatment was appropriate. ${ }^{6}$ They also considered percutaneous drainage of any paraspinal fluid collection to be an essential part of medical treatment and suggested that close monitoring with serial MRI is required to provide objective support for the decision to manage the patient non-operatively. However, the possibility of sudden neurological deterioration in patients receiving appropriate bactericidal antibiotics is well recognised. ${ }^{11}$ For this reason, we would suggest an additional guideline for the nonsurgical management of this conditionnamely, that such treatment only be undertaken in a hospital with full spinal surgical capabilities. In this way, emergency surgical decompression can be performed should sudden deterioration occur. Given that the reported incidence of SEA is two cases per 10000 hospital admissions, such a policy would not add significantly to the overall workload of a regional spinal surgery service. It 

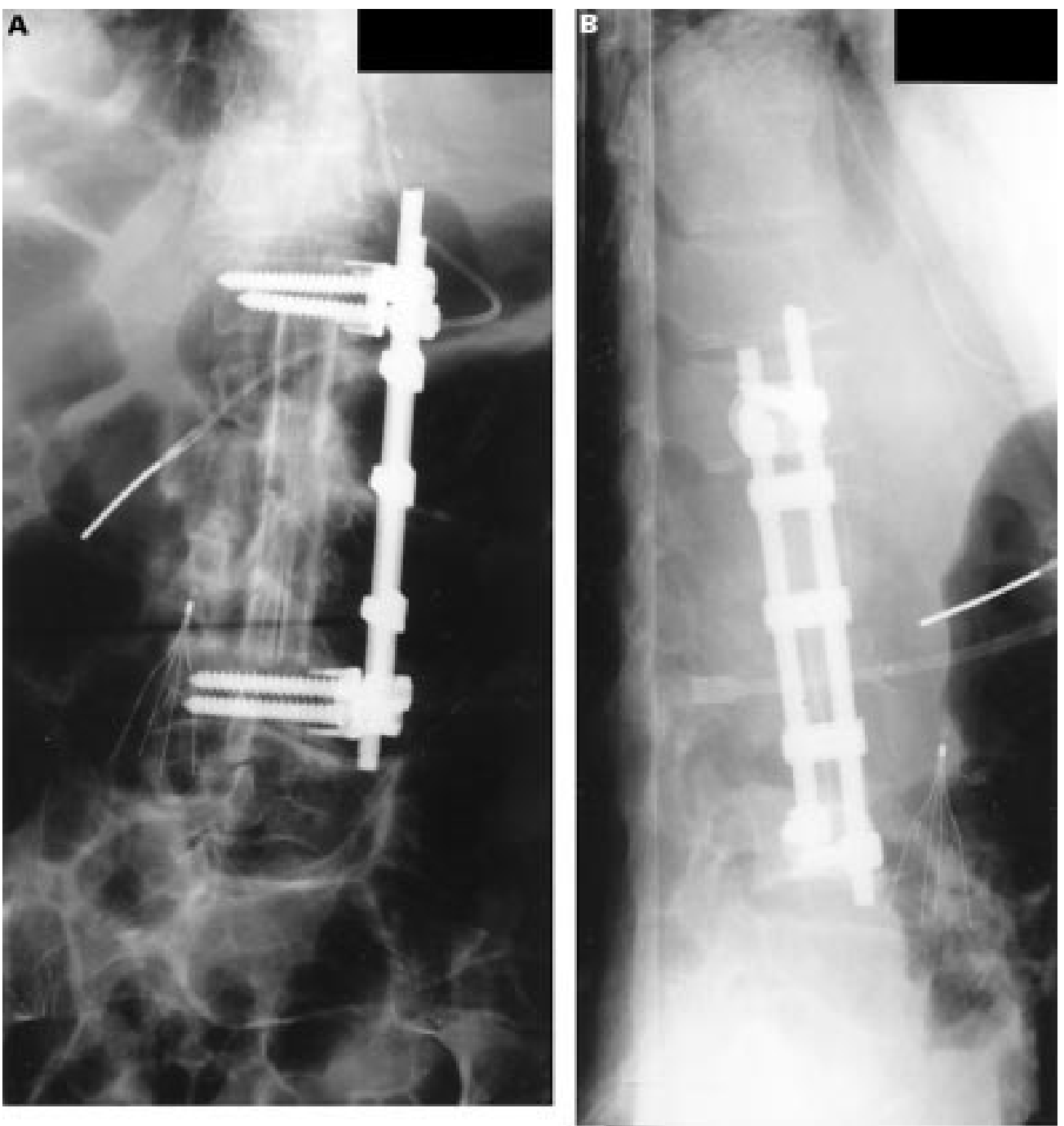

Figure 4 (A) Anteroposterior and (B) lateral radiographs after anterior decompression and stabilisation using two fibular strut grafts and Kaneda instrumentation. An umbrella caval filter and fine bore enteral feeding tube can be seen.

would seem reasonable to argue that the clinician responsible for monitoring the neurological status of these patients should work in close cooperation with the specialist who will be responsible for performing surgery should the need arise.

In the case we report, non-surgical management was continued in the face of a number of contraindications. The patient had persistent, severe pain which failed to improve, the temperature and blood indices (WCC, ESR) remained raised, spinal deformity became apparent, and there was clear neurological deterioration with the gradual onset of paraparesis. Finally, the duration of non-surgical treatment was inappropriate. The lack of significant clinical improvement after six weeks of intravenous antibiotics constitutes an obvious indication for more radical treatment - that is, surgical debridement of the anterior column of the spine. The decision by the general surgeon to perform a limited open debridement of the psoas collection did not deal with the source of the problem and undoubtedly contributed to the protracted clinical course in this case. The reintroduction of methotrexate treatment tipped the balance in depressing the patient's immune response, leading to the relapse.

SEA usually involves multiple levels and classically presents in elderly patients with some degree of immunocompromise. ${ }^{12}$ The reported mortality remains high. Non-surgical management on the grounds that "a considerable length of the vertebral column is affected" or because "the patient is too ill to withstand major surgery" is inappropriate. Rather, if the clinical condition fails to improve despite appropriate antibiotics for a known infecting organism, then the patient is too ill not to have surgery.

Several authors have proposed a prospective study of medical versus surgical management of SEA. ${ }^{811}$ However, we agree with Baker et al who suggested that such a study would be impractical because of the rarity of the condition and the diversity with which it presents. ${ }^{9}$ Also, the real risk of sudden deterioration in patients in the group not receiving surgical treatment renders such a trial problematic from an ethical perspective.

In conclusion, we recognise that medical management may be appropriate in certain 
Box 1 Indications for surgical management of spinal epidural abscess

- Persistent pyrexia, persistently raised WCC, ESR

- Failure to identify causative organism

- Persistent severe pain

- Spinal deformity or instability

- Deteriorating neurology (progression to Rankin stage III $)^{13}$

- MRI showing $>50 \%$ compression of thecal sac

- Lack of availability of serial MRI

- Lack of availability of emergency spinal surgery facilities

- Failure to resolve despite prolonged antibiotic treatment ( $>6$ weeks)

- Depressed host immune response

cases. However the widely agreed contraindications bear reiteration (box 1).

1 Smith AS, Blaser SI. Infections and inflammatory processes of the spine. Radiol Clin North Am 1991;17:773-94.
2 Walker AE. A history of neurological surgery. New York: Hafner, 1967.

3 Dandy WE. Abscesses and inflammatory tumors in the spinal epidural space. Arch Surg 1926;13:477-94.

4 Heusner AP. Non tuberculous spinal epidural infections. N Engl J Med 1948;239:845-54.

5 Del Curling O, Gower DJ, McWhorter JM. Changing concepts in spinal epidural abscess: a report of 29 cases. Neurosurgery 1990;27:185-92.

6 Baker AS, Ojemann RG, Swartz MN, Richardson EP. Spinal epidural abscess. N Engl J Med 1975;293:463-8.

7 Leys D, Lesoin F, Viaud C, Pasquier F, Rousseaux M, Jomin $\mathrm{M}$, et al. Decreased morbidity from acute bacterial spinal epidural abscess using computed tomography and nonsurgical treatment in selected patients. Ann Neurol 1985;17: 350-5.

8 Wheeler D, Keiser P, Rigamonti D, Keay S. Medical management of spinal epidural abscess: case report and review. Clin Infect Dis 1992;15:22-7.

9 Baker AS, Ojeman RG, Baker RA. To decompress or not to decompress spinal epidural abscess [editorial response]. Clin Infect Dis 1992;15:28-9.

10 Rea GL, McGregor JM, Miller CA, Miner ME. Surgical treatment of the spontaneous spinal epidural abscess. Surg Neurol 1992;37:274-9.

11 Khanna RK, Malik GM, Rock JP, Rosenblum LM. Spinal epidural abscess: evaluation of factors influencing outcome. Neurosurgery 1996;39:958-64.

12 Carragee EJ. Pyogenic vertebral osteomyelitis. J Bone Joint Surg Am 1997;79:874-80.

13 Rankin RM, Flothow PG. Pyogenic infection of the spinal epidural space. West J Surg Obstet Gynecol 1946;54: 320-3. 